\title{
Multispin dynamics of the solid-state NMR free induction decay
}

\author{
H. Cho, ${ }^{1}$ T. D. Ladd, ${ }^{2}$ J. Baugh, ${ }^{3}$ D. G. Cory, ${ }^{1}$ and C. Ramanathan ${ }^{1, *}$ \\ ${ }^{1}$ Department of Nuclear Science and Engineering, Massachusetts Institute of Technology, Cambridge, Massachusetts 02139, USA \\ ${ }^{2}$ Department of Applied Physics, Stanford University, Palo Alto, California 94305, USA \\ ${ }^{3}$ Institute for Quantum Computing, University of Waterloo, Waterloo, Ontario, Canada
}

(Received 24 January 2005; published 18 August 2005)

\begin{abstract}
We present a new experimental investigation of the NMR free induction decay (FID) in a lattice of spin-1/2 nuclei in a strong Zeeman field. Following a $\pi / 2$ pulse, evolution under the secular dipolar Hamiltonian preserves the coherence number in the Zeeman eigenbasis, but changes the number of correlated spins in the state. The observed signal is seen to decay as single-spin, single-quantum coherences evolve into multiple-spin coherences under the action of the dipolar Hamiltonian. In order to probe the multiple-spin dynamics during the FID, we measured the growth of coherence orders in a basis other than the usual Zeeman eigenbasis. This measurement provides the first direct experimental observation of the growth of coherent multiple-spin correlations during the FID. Experiments were performed with a cubic lattice of spins ( ${ }^{19} \mathrm{~F}$ in calcium fluoride) and a linear spin chain $\left({ }^{19} \mathrm{~F}\right.$ in fluorapatite). It is seen that the geometrical arrangement of the spins plays a significant role in the development of higher-order correlations. The results are discussed in light of existing theoretical models.
\end{abstract}

DOI: 10.1103/PhysRevB.72.054427

Solid-state NMR is an ideal test bed for studying coherent quantum dynamics in a large Hilbert space. In this work, we experimentally investigate the many-spin dynamics of the NMR free induction decay. The free induction decay (FID) is the response of the spin system following a $\pi / 2$ pulse. In a solid lattice of spin-1/2 nuclei in a strong magnetic field, this evolution is dominated by the secular dipolar Hamiltonian. This is a classic problem in spin dynamics and has been well studied since the early days of NMR. ${ }^{1-11}$

At the magnetic fields typically used in NMR experiments $(<20 \mathrm{~T})$, the spin system is highly mixed at room temperature, and its equilibrium state is represented by a thermal density matrix. If the external Zeeman field is much stronger than the internal dipolar fields of the sample, the normalized deviation density matrix in thermal equilibrium can be approximated as

$$
\hat{\rho}(0)=-\sum_{j} \hat{I}_{j z}
$$

Following a $\pi / 2$ pulse, the spins evolve under the secular dipolar Hamiltonian,

$$
\hat{H}_{\mathrm{int}}=\sum_{j<k} D_{j k}\left\{\hat{I}_{j z} \hat{I}_{k z}-\frac{1}{4}\left(\hat{I}_{j+} \hat{I}_{k-}+\hat{I}_{j-} \hat{I}_{k+}\right)\right\} .
$$

The strength of the dipolar coupling $D_{j k}$ between spins $j$ and $k$ is given by

$$
D_{j k}=\frac{\gamma^{2} \hbar^{2}}{r_{j k}^{3}}\left(1-3 \cos ^{2} \theta_{j k}\right),
$$

where $\gamma$ is the gyromagnetic ratio, $r_{j k}$ is the distance between spins $j$ and $k$, and $\theta_{j k}$ is the angle between the external magnetic field and internuclear vector $\vec{r}_{j k}$. Since the Hamiltonian is time independent, the formal solution of the Liouville-von Neumann equation yields the density matrix of the spin system at time $t$ following the pulse as
PACS number(s): 75.40.Gb, 76.60.-k, 82.56.-b

$$
\hat{\rho}(t)=e^{-i \hat{H}_{\text {int }} t / \hbar} \hat{\rho}(0) e^{i \hat{H}_{\text {int }} t / \hbar} .
$$

An exact solution to this many-body problem has not been found, but the equation can be expanded in a power series to examine the short time behavior of the system:

$$
\hat{\rho}(t)=\hat{\rho}(0)+\frac{i}{\hbar} t\left[\hat{\rho}(0), \hat{H}_{\mathrm{int}}\right]-\frac{t^{2}}{2 \hbar^{2}}\left[\left[\hat{\rho}(0), \hat{H}_{\mathrm{int}}\right], \hat{H}_{\mathrm{int}}\right]+\ldots .
$$

In an inductively detected NMR experiment (in which a coil is used to measure the average magnetization in the transverse plane), the observed signal is given by

$$
S(t)=\zeta\left\langle\hat{I}_{+}\right\rangle=\zeta \operatorname{Tr}\left\{\hat{I}_{+} \hat{\rho}(t)\right\},
$$

where $\hat{I}_{+}=\Sigma_{j}\left(I_{j x}+i I_{j y}\right)$ and $\zeta$ is a proportionality constant. The only terms in $\hat{\rho}(t)$ that yield a nonzero trace in the above equation and therefore contribute to the observed signal $S(t)$ are the single-spin angular momentum operators such as $\hat{I}_{j-}$, which are single-spin, single-quantum coherences. Singlequantum coherences are off-diagonal terms of the density matrix (in the Zeeman eigenbasis or the $z$ basis) connecting eigenstates with $\Delta m= \pm 1$ (corresponding to coherent superpositions of these eigenstates). Evaluating the commutators in Eq. (5),

$$
\begin{aligned}
\hat{\rho}(t)= & -\frac{1}{2} \sum_{j}\left(\hat{I}_{j+}+\hat{I}_{j-}\right)+\frac{3}{2} i t \sum_{j k} D_{j k}\left(-\hat{I}_{j z} \hat{I}_{k+}+\hat{I}_{j z} \hat{I}_{k-}\right) \\
& -\frac{3}{4} t^{2} \sum_{j k l} D_{l k} D_{j k}\left(\hat{I}_{j z} \hat{I}_{l z} \hat{I}_{k+}+\hat{I}_{j z} \hat{I}_{l z} \hat{I}_{k-}\right)+\cdots .
\end{aligned}
$$

Substituting Eq. (7) into Eq. (6), it can be seen that the observable magnetization decays during the evolution under $\hat{H}_{\text {int }}$ because single-spin, single-quantum coherence terms are 
transformed to unobservable multiple-spin, single-quantum coherence terms by the higher-order nested commutators. The $n$-th term in the expansion in Eq. (5) has $n$-spin correlations.

There has been much theoretical effort ${ }^{1-8}$ to predict the shape of the FID in calcium fluoride $\left(\mathrm{CaF}_{2}\right)$. Calcium fluoride is a standard test system for spin dynamics as the ${ }^{19} \mathrm{~F}$ (spin-1/2) nuclei are $100 \%$ abundant and form a simple cubic lattice. The main goal has been to reproduce the decay and beat pattern of the observed time-domain NMR signal. For example, Engelsberg and Lowe ${ }^{10}$ measured up to 14 moments of the FID in $\mathrm{CaF}_{2}$, and these were found to be in good agreement with theoretically calculated values for the second to eighth moments. The odd moments of the FID are zero, and the even moments are given by ${ }^{1}$

$$
M_{2 n}=\frac{(-1)^{n}}{\operatorname{Tr}\left\{\hat{I}_{x}^{2}\right\}} \operatorname{Tr}\{\underbrace{\left[\hat{H}_{\mathrm{int}},\left[\hat{H}_{\mathrm{int}},\left[\ldots,\left[\hat{H}_{\mathrm{int}}, \hat{,}_{x}\right] \ldots\right]\right]\right.}_{2 \mathrm{ntimes}} \hat{I}_{x}\} .
$$

Evaluating the nested commutators becomes increasingly challenging and the higher-order moments are difficult to calculate. However, it is these higher moments that characterize the many-spin correlations in the spin system. It can be seen that the $2 n$-th moment arises from the $(2 n+1)$-th term in the expansion in Eq. (5), which creates up to $(2 n+1)$ correlated spins. The main weakness of the moment method lies in the fact that the most important contribution to the value of the higher moments comes from the tails of the FID, which are acquired with the lowest signal-to-noise ratio (SNR) in typical FID measurements. ${ }^{3}$

In this paper, we use a modified multiple-quantum NMR technique ${ }^{14}$ to study multiple-spin dynamics during the FID. Standard multiple-quantum techniques ${ }^{15-18}$ encode coherence orders in the Zeeman eigenbasis (or $z$ basis), but coherence numbers are conserved under the secular dipolar Hamiltonian in this basis. ${ }^{19}$ In our experiment we encode multiplequantum coherences in the $x$ basis. The dipolar Hamiltonian in the $x$ basis is

$$
\begin{aligned}
\hat{H}_{\mathrm{int}}^{x}= & -\frac{1}{2} \sum_{j<k} D_{j k}\left\{\hat{I}_{j x} \hat{I}_{k x}-\frac{1}{4}\left(\hat{I}_{j+}^{x} \hat{I}_{k-}^{x}+\hat{I}_{j-}^{x} \hat{I}_{k+}^{x}\right)\right\} \\
& -\frac{3}{8} \sum_{j<k} D_{j k}\left(\hat{I}_{j+}^{x} \hat{I}_{k+}^{x}+\hat{I}_{j-}^{x} \hat{I}_{k-}^{x}\right),
\end{aligned}
$$

and no longer conserves coherence order in this basis (we use the superscript $x$ to denote that the raising and lowering operators are defined in the $x$ basis; these operators are otherwise assumed to be expressed in the $z$ basis). The coherence orders are encoded by a collective rotation about the $x$ axis (which is the effective quantizing axis in this basis). Transforming the density matrix shown in Eq. (7) into the $x$ basis yields

$$
\begin{aligned}
\hat{\rho}^{x}(t)= & -\sum_{j} \hat{I}_{j x}-\frac{3}{4} i t \sum_{j k} D_{j k}\left(\hat{I}_{j+}^{x} \hat{I}_{k+}^{x}-\hat{I}_{j-}^{x} \hat{I}_{k-}^{x}\right) \\
& +\frac{3}{8} t^{2} \sum_{j k l} D_{l k} D_{j k}\left(\hat{I}_{j+}^{x} \hat{I}_{l+}^{x} \hat{I}_{k x}-\hat{I}_{j+}^{x} \hat{I}_{l-}^{x} \hat{I}_{k x}-\hat{I}_{j-}^{x} \hat{I}_{l+}^{x} \hat{I}_{k x}\right. \\
& \left.+\hat{I}_{j-}^{x} \hat{I}_{l-}^{x} \hat{I}_{k x}\right)+\ldots .
\end{aligned}
$$

(a) $z$-basis

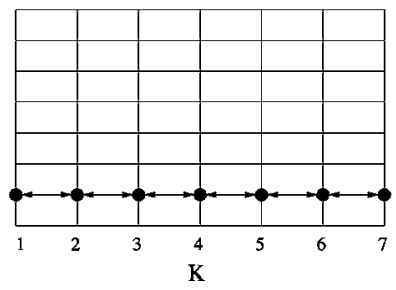

(b) $x$-basis

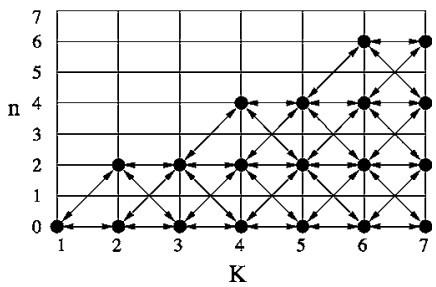

FIG. 1. Projection of Liouville space onto the two-dimensional plane spanned by $K$ and $n$, showing the dynamics of the FID in (a) the Zeeman eigenbasis, and (b) the $x$ basis. The arrows show the allowed paths in each case.

From Eq. (10), it can be seen that, starting from an initial $I_{x}$ state, even-order multiple-quantum coherences are created in the $x$ basis. It is possible to generate only odd-order coherences using a $y$-basis encoding for the same initial state.

It is useful to consider the dipolar evolution of this highly mixed state using the Liouville space formulation for multiple-quantum dynamics suggested previously. ${ }^{17}$ The density operator in Liouville space can represented as

$$
\hat{\rho}(t)=\sum_{K=0}^{N} \sum_{n=-K}^{K} \sum_{p} g_{K n p}(t) \hat{P}_{K n p},
$$

where $\hat{P}_{K n p}$ represents a basis operator that is a product of $K$ single-spin angular momentum operators, $n$ is the coherence order of the operator, and $p$ is a label that identifies a particular configuration of spins having the same $K$ and $n$. The selection rules for the dipolar Hamiltonian in the Zeeman basis are given by

$$
\Delta K= \pm 1, \quad \Delta n=0 .
$$

A projection of Liouville space onto the two-dimensional plane spanned by $K$ and $n$ is shown in Fig. 1(a). Following a $\pi / 2$ pulse, the trajectory in the Zeeman basis is indicated by the arrows (only positive coherences are shown here; the evolution is perfectly symmetric for negative $n$ ). Increasing numbers of spins are correlated following evolution under the dipolar Hamiltonian, but the coherence number does not change. Figure 1(b) shows the same evolution in the $x$ basis, where the selection rules are

$$
\Delta K= \pm 1, \quad \Delta n=0, \pm 2 .
$$

Starting from an initial $I_{x}$ state $(K=1, n=0)$, only even-order coherences are observed. In this paper we characterize the growth of these coherences.

The pulse sequence used in this experiment is shown in Fig. 2. After an initial $\pi / 2$ pulse, multiple-spin, singlequantum states in the Zeeman basis are created during evolution under the secular dipolar Hamiltonian, as described in Eq. (7). A $\phi \hat{I}_{x}$ rotation encodes coherence orders in the $x$ basis, and a magic-echo sequence ${ }^{12}$ is used to refocus the multiple-spin terms back to observable single-spin, singlequantum coherence terms. The $\phi \hat{I}_{x}$ rotation is obtained by applying two $\pi / 2$ pulses, with phases $y+\phi$ and $\bar{y}$, which results in the propagator $\exp \left(i \phi \hat{I}_{z}\right) \exp \left(i \phi \hat{I}_{x}\right)$. The initial $\pi / 2$ 


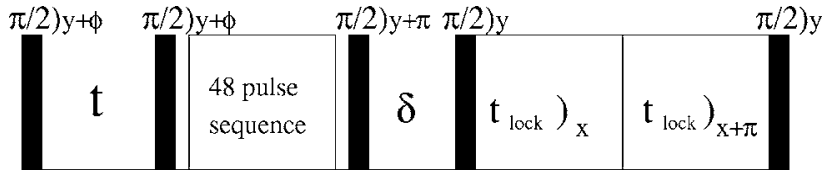

FIG. 2. The pulse sequence used in this experiment. $t$ is the evolution time under the secular dipolar Hamiltonian. The 48-pulse sequence was used to suppress the evolution of the internal Hamiltonian during the $x$-basis encoding step. A magic-echo sequence was used to reverse the dipolar evolution. A delay ( $\delta=10 \mu \mathrm{s})$ was inserted before the magic echo to push the echo out, in order to minimize any pulse transients and receiver dead-time effects. The duration of the spin-locking time in the magic-echo sequence is $2 t_{\text {lock }}$, where $t_{\text {lock }}=t+2 \delta+3 \mu \mathrm{s}$.

excitation pulse is also phase shifted by $\phi$ to cancel out the rotation about $\hat{I}_{z}$. Since it is difficult to apply back-to-back $\pi / 2$ pulses without a delay between them (without introducing phase transients or allowing some dipolar evolution during the pulses), an evolution-suspension sequence needs to be used in between the two $\pi / 2$ pulses. In this experiment we use a previously described 48-pulse evolution-suspension sequence. ${ }^{13}$

The experiments were performed at room temperature at $2.35 \mathrm{~T}\left(94.2 \mathrm{MHz},{ }^{19} \mathrm{~F}\right)$, using a Bruker Avance spectrometer and home-built probe. The samples used were a $1 \mathrm{~mm}^{3}$ single crystal of $\mathrm{CaF}_{2}$ with $T_{1} \sim 7 \mathrm{~s}$, and a crystal of fluorapatite (FAp) with $T_{1} \sim 200 \mathrm{~ms}$. The FAp crystal is a mineral crystal specimen from Durango, Mexico. Such natural crystals are expected to have a number of interruptions in the spin chains as well as a variety of other defects, ${ }^{20}$ as indicated by the short $T_{1}$. All experiments were conducted on resonance. High-power $0.5 \mu \mathrm{s} \pi / 2$ pulses were used for the 48-pulse evolution suspension sequence, while lower-power $1.5 \mu \mathrm{s} \pi / 2$ were used during the magic-echo sequence, as this sequence is more susceptible to phase-transient errors. The phase $\phi$ was incremented from 0 to $4 \pi$ with $\Delta \phi$ $=\pi / 32$ to encode up to 32 quantum coherences for every experiment. A fixed time point corresponding to the maximum intensity signal was sampled for each $\phi$ value and Fourier transformed with respect to $\phi$ to obtain the coherence order distribution at each dipolar evolution time $t$.

Figure 3 shows the coherence-order distribution observed for $\mathrm{CaF}_{2}$ at various time points during the FID. At short times, the maximum coherence order $\left(n_{\max }\right)$ corresponds to the maximum number of correlated spins $\left(K_{\max }\right.$, i.e., $g_{K n p}$ $=0$ for $\left.K>K_{\max }\right)$. At longer times, the maximum coherence order observed in the experiment sets the lower limit of the size of the spin correlation, since the SNR of higher-order coherences might be too low to be observed.

Figure 4 shows the growth of the different coherence orders in $\mathrm{CaF}_{2}$ during the FID. The inset shows the initial oscillation between the zero- and double-quantum coherences at short times (which corresponds to single- and twospin correlations, respectively) due to the resolved nearestneighbor coupling at the [100] and [110] directions. This oscillation may be theoretically understood by considering the time development of an isolated pair of spins under the secular dipolar Hamiltonian (in the $x$ basis):

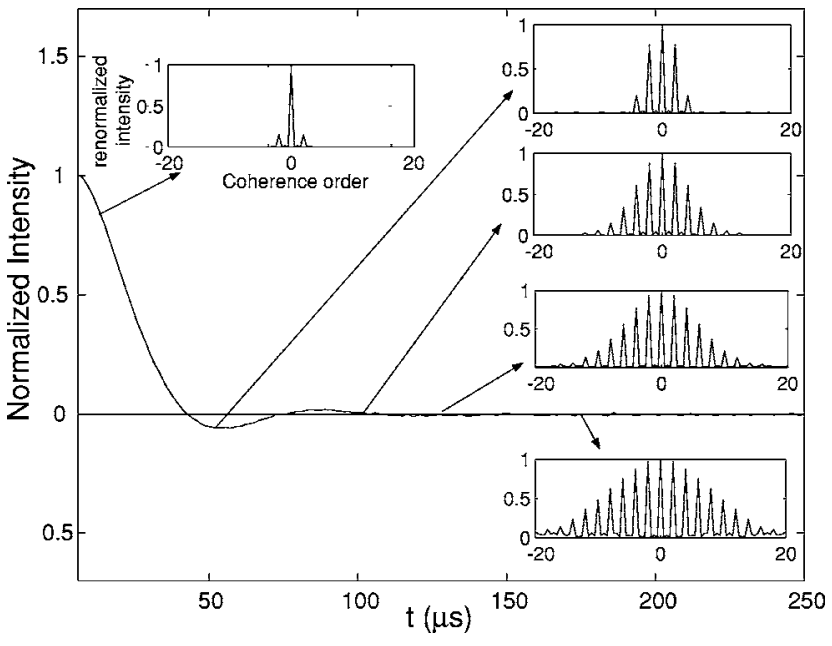

FIG. 3. The $x$-basis coherence order distribution at various time points under the evolution of the secular dipolar Hamiltonian in $\mathrm{CaF}_{2}$. The multiple-quantum intensities have been renormalized to set the intensity of the zero-quantum term to one in each case.

$$
\rho(t)=\frac{1}{2} \cos \left(\frac{3 D t}{2}\right)\left(I_{1 x}+I_{2 x}\right)-\frac{i}{4} \sin \left(\frac{3 D t}{2}\right)\left(I_{1+}^{x} I_{2+}^{x}-I_{1-}^{x} I_{2-}^{x}\right),
$$

where $D$ is the strength of the pairwise coupling. In an extended spin system, this oscillation is rapidly damped by leakage from isolated pairs to higher-order correlations. ${ }^{21}$ The higher-order coherences $(n \geqslant 4)$ are seen to follow a sigmoidal growth curve. The higher-order coherences develop later in time, and this progressive growth leads to a saturation of the intensities of the lower-order coherences, consis-

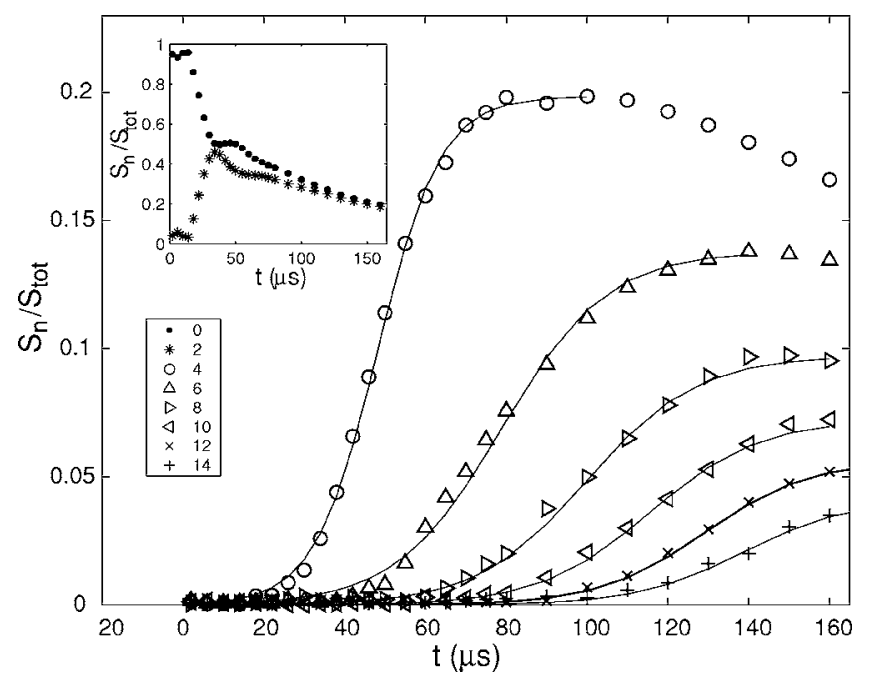

FIG. 4. The growth of multiple-spin correlations during the FID, showing the sigmoidal fit to the initial growth data of each $x$-basis coherence order $(n \geqslant 4)$, along $\sim[110]$ direction in $\mathrm{CaF}_{2}$. The inset shows the dynamics of $n=0$ and 2 . The intensity of the signal for each coherence order $S_{n}$ is normalized with respect to the total signal $S_{\text {tot }}$, in order to compensate for imperfect refocusing of the dipolar interaction. 


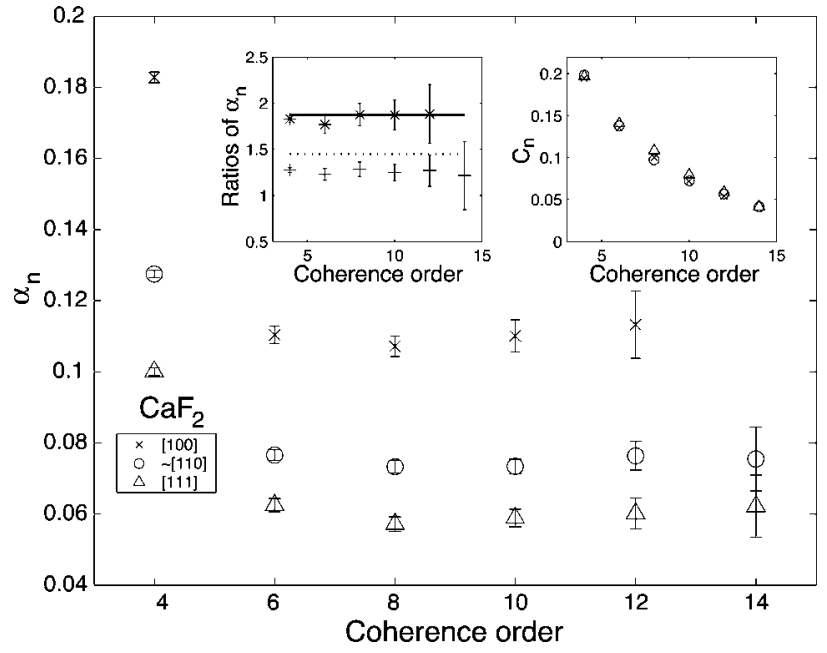

FIG. 5. The values of $\alpha_{n}$ for different orientations in $\mathrm{CaF}_{2}$. The left inset shows the ratios $\alpha_{[100]} / \alpha_{[111]}(*)$ and $\alpha_{\sim[110]} / \alpha_{[111]}(+)$. The calculated ratios of the mean dipolar-coupling strength, obtained by averaging over 26 nearest neighbors, [100]/[111] (solid line) and $\sim[110] /[111]$ (dotted line) are also shown. The right inset show the values of $C_{n}$ 's for a different orientation in $\mathrm{CaF}_{2}$.

tent with the model in Fig. 1. As $t$ increases, imperfect refocusing of the dipolar evolution under the magic-echo sequence results in a decay of the observed signal. In order to remove this decay the intensity for each coherence order is normalized with respect to the total signal measured at that evolution time.

We have fit the initial growth of each coherence order to the following sigmoidal function:

$$
S_{n}(t)=\frac{C_{n}}{1+e^{-\alpha_{n}\left(t-t_{n}^{\text {onset }}\right)}} .
$$

In Fig. 5 we plot the variation of $\alpha_{n}$ and $C_{n}$ as a function of coherence order $n$. The parameter $\alpha_{n}$ represents the underlying rate at which the different coherence orders are transformed, and should be dominated by the strength of the dipolar couplings involved. It is seen that $\alpha_{n}$ does not vary with $n$, suggesting that the near-neighbor interactions dominate the dynamics here. The mean dipolar-coupling strength depends on the crystal orientation, so we measured $\alpha_{n}$ with the crystal oriented along the [111], [110], and [100] directions with respect to the external field. The mean dipolarcoupling strength can be estimated by summing $\left|D_{1 j}\right|$ over the 26 nearest-neighbor spins for each crystal orientation. For a simple cubic lattice, the ratio of these means is 1:1.45:1.87 for [111], [110], and [100], in good agreement with the ratios of $\alpha_{n}$ shown in the figure. The values of $C_{n}$ are seen to decrease as the coherence number increases, independently of the crystal orientation. This is expected as the total polarization is conserved, and the signal is progressively spread over increasingly larger regions of the system Hilbert space.

Figure 6 shows the onset time $t_{n}^{\text {onset }}$ of each of the $x$-basis coherences for different orientations of the crystal. Physically, the onset time corresponds to the time required for a

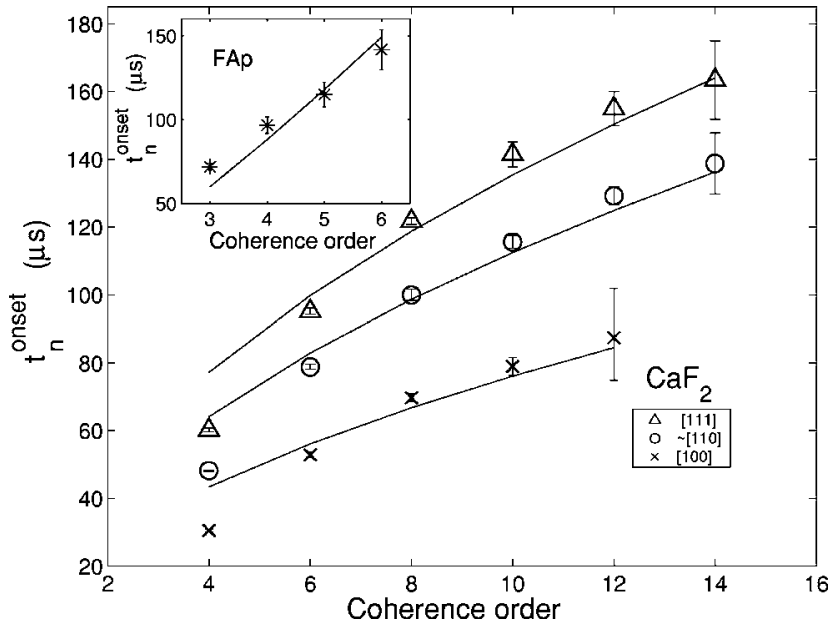

FIG. 6. The onset time of multiple-spin correlations for different crystal orientations in $\mathrm{CaF}_{2}$. The inset shows the onset times for the FAp sample. Odd coherence orders for the FAp sample were obtained using a $y$-basis encoding on the same initial state. The continuous lines represent the best fits of Eq. (20) to the data, assuming that the same equation is valid for the coherence number as well.

specific coherence order to become observable in the experiment. While a first glance at Eq. (7) would seem to suggest that higher-order correlations should develop as $t^{n}$, it is the geometry of the spin system (the values of $D_{i j}$ ) that ultimately determines the rate at which the spin correlations grow. The onset times depend on the rate at which the correlations are spreading through the spin system, which in turn depends on the value of the dipolar couplings. Thus, the rate is expected to be fastest (shorter onset time), with the crystal oriented along the [100] direction and slowest (longer onset time) for the crystal oriented along the [111] direction, in agreement with the experimental data. The variation of onset times with coherence order is also observed to depend on the dimensionality of the spin system. The variation is sublinear in the cubic $\mathrm{CaF}_{2}$ system and displays an approximate $n^{2 / 3}$ dependence. The inset in Fig. 6 shows that the onset times obtained for FAp, the quasi-one-dimensional spin system, vary linearly with coherence number, in marked contrast to the results from $\mathrm{CaF}_{2}$.

A variety of models have been proposed to describe the dynamics under a multiple-quantum Hamiltonian. The most commonly used model involves a random walk among the components of the Liouville-space basis set $\hat{P}_{\text {Knp }}$, subject to the selection rules of the multiple-quantum Hamiltonian. ${ }^{17}$ The model replaces the Liouville-von Neumann equation by a set of coupled rate equations with exponential solutions,

$$
\frac{d}{d t} \mathbf{g}=\mathbf{R} \cdot \mathbf{g},
$$

where the vector $\mathbf{g}$ contains the coefficents $g_{K n p}$. All possible configurations that contribute to a particular coherence are assumed to be present in equal measure, and the resulting growth of the spin system is described by a hopping procedure between the allowed points on the lattice (shown in Fig. 1). Under this assumption, the hopping rates are solely de- 
termined by the degeneracies of the coupled states. The model thus eliminates any oscillatory solutions and precludes the possibility of quantum interference effects playing a role in the evolution. The model also ignores the geometrical arrangement of the spin system and the specific distribution of dipolar-coupling coefficients responsible for driving the evolution. All spin systems display a universal growth kinetics, as long as the dynamics are scaled by a lattice parameter that represents the mean dipolar-coupling strength of the system, similar to the parameter $\alpha_{n}$ obtained above. A numerical solution of the coupled equations for the multiple-quantum evolution was observed to yield sigmoidal growth curves for the various coherence orders. ${ }^{17}$ Limitations to this model have been discussed elsewhere. ${ }^{22}$

Munowitz and Mehring ${ }^{11}$ used this model to numerically simulate the growth of multispin dynamics of the FID in a 21 -spin system. In order to track the development of correlations among the spins, they defined an induction time $t_{K}$ over which a particular $K$-spin coherence reaches half its maximum value. This parameter is very similar to the experimentally measured onset times of the different coherence orders described here. Figure 7 of Ref. 11 shows the variation of induction time with the number of correlated spins. The numerical results show that the variation of the induction time is sublinear for small numbers of correlated spins $(<10)$, in agreement with the experimental data. For larger numbers of correlated spins, however, there is a marked deviation from the sublinear behavior, as the effects of the finite system size (21 spins) begin to manifest themselves in the simulations. The number of correlated spins would have to approach the number of spins in the sample $\left(\approx 10^{21}\right)$ before such effects would be observed experimentally. While providing some insight into the growth of the dynamics for the cubic spin systems, it is seen that the model does not do a very good job at describing the one-dimensional spin system. It is in this situation that the spin geometry plays a dominant role.

Gleason and co-workers have proposed an alternate model to describe the growth of spin correlations that emphasizes the geometrical ordering of spins. ${ }^{23}$ By aggregating over the different configurations and coherence orders, i.e., summing over $n$ and $p$ in Eq. (11), the density operator $\hat{\rho}$ is expressed as a sum of terms with spin number $K$ and coefficients $g_{K}$,

$$
\hat{\rho}(t)=\sum_{K=0}^{N} g_{K}(t) \hat{P}_{K},
$$

where $g_{K} \hat{P}_{K}=\sum_{n p} g_{K n p} \hat{P}_{K n p}$. Essentially, this model assumes a single effective $K$-spin operator that incorporates all the possible spin and spatial configurations of the $K$ spins. The resulting model for spin propagation through a lattice yields a differential equation for the coefficients $g_{K}(t)$ of the form

$$
\frac{d}{d t} g_{K}=-i\left(W_{K-1}^{f} g_{K-1}+W_{K+1}^{r} g_{K+1}\right),
$$

where the rate constants $W^{f}$ and $W^{r}$ correspond to the forward and reverse rates, respectively. Under the assumption that the spatial grouping of the $K$ spins is continuous, and that only the nearest-neighbor couplings are important, the forward rate (and equivalently the reverse rate) can be expressed as $W_{K}^{f} \propto D n_{n} n_{s}$, where $D$ is the strength of the nearest neighbor coupling, $n_{s}$ is the number of spins on the surface of the spatial grouping, and $n_{n}$ is the number of neighboring spins coupled to each spin. New spins are added on the surface of the correlated spin cluster. While $n_{n}$ is a constant, the term $n_{s}$ would differ significantly for spin systems of different dimensionalities, and can be expressed as $n_{s} \propto K^{1-1 / d}$, where $d$ is the dimensionality of the spin system. For a linear spin chain, $d=1$ and $n_{s}$ is independent of $K$, while for a cubic spin system, $d=3$ and $n_{s} \propto K^{2 / 3}$. While this model does not discuss coherence order, the dimensional dependence does agree with the experimental results, if the onset time characterizes the effective rate constant. In the limit of large $K$, the rate constants $W_{K-1}^{f} \approx W_{K+1}^{r}$, and the coefficients $g_{K}(t)$ are approximately given by

$$
g_{K}(t) \propto i^{K-1}\left[\tanh \left(\beta t K^{-1 / d}\right)\right]^{K},
$$

where $\beta$ is proportional to the mean dipolar-coupling strength. With an appropriate choice of normalization, the intensities $\left|g_{K}(t)\right|^{2}$ obtained from Eq. (19) show the same sigmoidal growth characteristics of the multiple quantum coherence intensities shown in Fig. 4. An onset time can be obtained from Eq. (19) by setting $\left[\tanh \left(\beta t K^{-1 / d}\right)\right]^{2 K}=1 / 2$, yielding

$$
t_{1 / 2}=\frac{\eta}{\beta} K^{1 / d} \operatorname{arctanh}\left(2^{-1 / 2 K}\right),
$$

where $\eta$ is a constant scaling factor. Figure 6 shows the best fit of Eq. (20) to the experimental data, assuming that the model holds true for coherence number as well. It is seen that there is excellent agreement at larger values of $n$ for the cubic $\mathrm{CaF}_{2}$ system. The values of $\eta / \beta$ obtained from the fit are 31.19 in the [111] direction, 25.74 in the [110] direction, and 17.42 in the [001] direction. Their inverses are in the ratio 1:1.48:1.79 for [111]:[110]:[100], which is in excellent agreement with the theoretically calculated values shown earlier. For the linear FAp system, Eq. (20), which is linear for large $K$, is observed to be weakly superlinear at these small values of $K .^{24}$

The constancy of $\alpha_{n}$ in the sigmoidal plots in Fig. 4 and the good agreement observed between the observed onset times and Eq. (20) indicate that the spin dynamics are dominated by the nearest-neighbor interactions in this regime. This is not surprising, as we are still operating in the shorttime regime. Higher-order spin processes, if significant, would be expected to manifest themselves at later times, leading to a deviation from the simple model behavior described above.

In conclusion, we have presented a new experimental method to characterize the multispin dynamics of the solidstate NMR free induction decay. The initial creation of coherences were observed to follow a sigmoidal growth curve, 
with the onset times characterizing the dynamics of the spin system. These dynamics in turn were critically dependent on the geometrical arrangement of the spins, as expected.
The authors thank D. Greenbaum, P. Capperallo, and T. S. Mahesh for helpful discussions, and the NSF, ARO, and DARPA DSO for financial support.
*Author to whom correspondence should be addressed. Electronic address: sekhar@mit.edu

${ }^{1}$ J. H. van Vleck, Phys. Rev. 74, 1168 (1948).

${ }^{2}$ I. J. Lowe and R. E. Norberg, Phys. Rev. 107, 46 (1957).

${ }^{3}$ A. Abragam, Principles of Nuclear Magnetism (Clarendon, Oxford, 1961).

${ }^{4}$ A. A. Ludin and B. N. Provotorov, Phys. Lett. 55A, 426 (1976).

${ }^{5}$ F. Lado, J. D. Memory, and G. W. Parker, Phys. Rev. B 4, 1406 (1971).

${ }^{6}$ B. V. Fine, Phys. Rev. Lett. 79, 4673 (1997).

${ }^{7}$ A. A. Nevzorov and J. H. Freed, J. Chem. Phys. 112, 1425 (2000).

${ }^{8}$ A. A. Nevzorov and J. H. Freed, J. Chem. Phys. 115, 2401 (2001)

${ }^{9}$ M. Engelsberg, I. J. Lowe, and J. L. Carolan, Phys. Rev. B 7, 924 (1973).

${ }^{10}$ M. Engelsberg and I. J. Lowe, Phys. Rev. B 10, 822 (1974).

${ }^{11}$ M. Munowitz and M. Mehring, Chem. Phys. 116, 79 (1987).

${ }^{12}$ W.-K. Rhim, A. Pines, and J. S. Waugh, Phys. Rev. B 3, 684 (1971).

${ }^{13}$ D. G. Cory, J. B. Miller, and A. N. Garroway, J. Magn. Reson. (1969-1992) 90, 205 (1990).

${ }^{14}$ C. Ramanathan, H. Cho, P. Capperallo, G. S. Boutis, and D. G. Cory, Chem. Phys. Lett. 369, 311 (2003).

${ }^{15}$ Y.-S. Yen and A. Pines, J. Chem. Phys. 78, 3579 (1982).
${ }^{16}$ J. Baum, M. Munowitz, A. N. Garroway, and A. Pines, J. Chem. Phys. 83, 2015 (1985).

${ }^{17}$ M. Munowitz, A. Pines, and M. Mehring, J. Chem. Phys. 86, 3172 (1987).

${ }^{18}$ D. Suter, S. B. Liu, J. Baum, and A. Pines, Chem. Phys. 114, 103 (1987).

${ }^{19}$ The conservation of coherence order under the dipolar Hamiltonian is a consequence of the truncation of the nonsecular components of the dipolar interaction in a strong Zeeman field. The nonsecular components can change coherence orders by \pm 1 or \pm 2 .

${ }^{20}$ G. Cho and J. P. Yesinowski, J. Phys. Chem. 100, 15716 (1996).

${ }^{21}$ C. Ramanathan, S. Sinha, J. Baugh, T. F. Havel, and D. G. Cory, Phys. Rev. A 71, 020303(R) (2005).

${ }^{22}$ S. Lacelle, Adv. Magn. Opt. Reson. 15, 173 (1991).

${ }^{23}$ D. H. Levy and K. K. Gleason, J. Phys. Chem. 96, 8125 (1992).

${ }^{24}$ Examining Eq. (18), we see that the initial growth of $g_{K}$ is dominated by $W_{K-1}^{f}$ as $g_{K+1}=0$. Thus the incremental time to go from a $(K-1)$-spin system to a $K$-spin system is inversely proportional to $W_{K-1}^{f}$. Applying this recursively, we obtain a general expression for the onset time $t_{K}=\eta H_{K-1}^{1-1 / d}$, where $\eta$ is a constant and $H_{n}^{r}$ is a generalized harmonic number of order $r$. The fits obtained with this equation are also in good agreement with the experimental data, and appear to be functionally identical to the Eq. (20) in the range of large $K$. 